Diabetes Obes Metab. Author manuscript; available in PMC 2016 September 01.

Published in final edited form as:

Diabetes Obes Metab. 2015 September ; 17(0 1): 6-11. doi:10.1111/dom.12514.

\title{
Molecular components of the circadian clock in mammals
}

\author{
Joseph S. Takahashi ${ }^{1,2,3}$ \\ ${ }^{1}$ Howard Hughes Medical Institute, University of Texas Southwestern Medical Center, Dallas, TX \\ 75390, USA \\ ${ }^{2}$ Department of Neuroscience, University of Texas Southwestern Medical Center, Dallas, TX \\ 75390, USA
}

\begin{abstract}
The circadian clock mechanism in animals involves a transcriptional feedback loop in which the bHLH-PAS proteins CLOCK and BMAL1 form a transcriptional activator complex to activate the transcription of the Period and Cryptochrome genes, which in turn feed back to repress their own transcription. In the mouse liver, CLOCK and BMAL1 interact with the regulatory regions of thousands of genes, which are both cyclically and constitutively expressed. The circadian transcription in the liver is clustered in phase and this is accompanied by circadian occupancy of RNA polymerase II recruitment and initiation. These changes also lead to circadian fluctuations in histone $\mathrm{H} 3$ lysine 4 trimethylation ( $\mathrm{H} 3 \mathrm{~K} 4 \mathrm{me} 3)$ as well as $\mathrm{H} 3$ lysine9 acetylation (H3K9ac) and $\mathrm{H} 3$ lysine27 acetylation (H3K27ac). Thus, the circadian clock regulates global transcriptional poise and chromatin state by regulation of RNA polymerase II.
\end{abstract}

\section{Keywords}

circadian clock; Clock gene; transcription; ChIP-seq; RNA-seq; RNA polymerase II; histone modifications; epigenetics; chromatin

\section{Introduction}

My laboratory is focused on understanding the molecular mechanism of circadian clocks in mammals and we have used mouse genetics as a tool for discovery of the critical genes involved in the generation of circadian rhythms of mammals [1,2]. The discovery of the Clock gene using forward genetic screens and positional cloning [3-5], and the identification of BMAL1 as the heterodimeric partner of CLOCK [6], led to idea that the CLOCK:BMAL1 transcriptional activator complex was upstream of the Period and Cryptochrome genes whose gene products then repressed CLOCK:BMAL1 to form an autoregulatory transcriptional feedback loop [7]. Since the identification of these "core circadian clock genes" (i.e., Clock, Bmall, Per1, Per2, Cryl and Cry2), additional feedback loops driven by CLOCK:BMAL1 such as the loop involving Rev-erba to repress Bmall

\footnotetext{
${ }^{3}$ Correspondence: University of Texas Southwestern Medical Center, 5323 Harry Hines Blvd., NA4.118, Dallas, TX 75390-9111, USA, joseph.takahashi@utsouthwestern.edu.

Conflict of Interest Statement

J.S.T. is a co-founder and SAB member of Reset Therapeutics, Inc.
} 
transcription have been described [8]. In addition, the regulation of the stability of the PER and CRY proteins by specific E3 ubiquitin ligase complexes has been found to be important for determining the periodicity of the circadian oscillation [9-13]. Together, this work has led to a description of a model of the circadian clock in mammals (Figure 1).

With the discovery and cloning of clock genes came the realization that their expression was ubiquitous [14]. We now accept that clock genes are housekeeping genes and are expressed in essentially all cells. What was perhaps even more surprising was the observation using circadian gene reporter technology that essentially every peripheral organ system and tissue has the capacity to express autonomous circadian rhythms [15]. Thus the ubiquitous expression of clock genes is a reflection of the ubiquitous capacity for most tissues and cells to express circadian oscillations. These distributed circadian oscillators are cell autonomous and can function independently of the central clock located in the suprachiasmatic nucleus (SCN) [15-17]. The realization that the body is composed of a multitude of cell-autonomous clocks has raised a number of questions concerning the organization of the clock system and the role of the SCN clock in "circadian organization." Elsewhere, we have also explored the role of the SCN as a master pacemaker to synchronize peripheral oscillators [15,18-22], as well as the role of intercellular coupling in robustness of the SCN oscillator [20,23-25].

\section{Transcriptional Architecture and Chromatin Dynamics of the Clock}

To define the cis-acting targets of the core circadian transcriptional regulators, we used chromatin immunoprecipitation followed by sequencing (ChIP-seq) to locate DNA binding sites for BMAL1, CLOCK, NPAS2, PER1, PER2, CRY1 and CRY2 in vivo in murine liver at 6 times during the circadian cycle. Figure 2 shows a browser view of the $D b p$ locus, a major target gene of CLOCK-BMAL1 [26]. The activators BMAL1, CLOCK and NPAS2 bind in a cyclic manner between CT0-12 (CT=circadian time; CT0 is the beginning of the subjective day; CT12 is the beginning of the subjective night) at three locations in the promoter, intron 1 and intron 2. PER1, PER2 and CRY2 bind the same sites with an opposite phase at CT12-20. CRY1 exhibits a third pattern that peaks at CT0.

In genome-wide analysis, CLOCK and BMAL1 bind to over 4,600 and 5,900 sites, respectively, corresponding to 3,000 unique genes [27]. The repressors CRY1 and CRY2 bind to significantly more sites and many thousands of these sites are independent of CLOCK:BMAL1 and reveal DNA binding motifs for nuclear receptors [27] including the glucocorticoid receptor consistent with recent work [28]. To examine functional readouts we used whole transcriptome RNA-seq to profile cycling genes in the liver using samples taken every 4 hours over 48 hours [27]. Using the intron RNA signal as a proxy for pre-mRNA, we found $~ 1300$ cycling genes, and surprisingly, they are clustered in time with a peak at CT15 (Figure 3). To explore the possible origins of the global rhythms in nascent transcription, we analyzed the genome-wide occupancy of RNA polymerase II (RNAPII) as a function of the circadian cycle. The large subunit of RNAPII contains a C-terminal domain (CTD) that is modified at various stages of transcription [29, 30]. RNAPII is recruited into the pre-initiation complex with a hypophosphorylated CTD that is recognized by the 8 WG16 antibody [31]. Again to our surprise, we find RNAPII-8WG16 occupancy is highly circadian across the genome in the liver with a peak at CT14.5, which precedes the intron 
RNA peak by 0.5 hours (Figure 3). Initiation of RNAPII involves phosphorylation on serine 5 (Ser5P) on the CTD of RNAPII and is recognized by the 3E8 antibody [32]. We find that RNAPII-Ser5P occupancy is also circadian with over 13,000 sites that are significant for cycling. The timing of RNAPII-Ser5P peaks at CT0 and coincides with the peak of CRY1. At this time we find an association of CRY1, CLOCK, BMAL1 and RNAPII-Ser5P binding sites, suggesting that CLOCK:BMAL1 can recruit and initiate RNAPII but CRY1 represses the complex leading to a "poised" state.

Given the genome-wide circadian rhythms of RNAPII occupancy, we assessed chromatin states associated with transcription initiation and elongation during the circadian cycle. Figure 4 shows a browser view of six histone modifications that are characteristic of promoters, enhancers and transcription elongation [33-39]. Histone H3K4me3, H3K9ac and $\mathrm{H} 3 \mathrm{~K} 27 \mathrm{ac}$ are enriched at promoters and show robust circadian rhythms in occupancy at the $D b p$ gene. When examined across the genome, we find that circadian rhythms in RNAPII occupancy as well as histone H3K4me3, H3K9ac and H3K27ac modifications occur in the majority of expressed genes even in cases where cycling RNA cannot be detected. Thus a third surprise in this work is the observation that chromatin states are being modulated in a circadian manner across the genome in the liver.

\section{Concluding Thoughts}

What accounts for these genome-wide circadian rhythms in RNAPII occupancy and histone modifications? Examination of the relationship between circadian transcription factor occupancy and gene expression shows that approximately $90 \%$ of genes bound by these factors are expressed whereas only 1-5\% of unexpressed genes are similarly bound [27].

These results demonstrate that gene expression per se rather than rhythmic gene expression is tightly correlated with circadian transcription factor binding. Rhythmic circadian transcription factor occupancy in turn could then be responsible for RNAPII recruitment and initiation on a genome-wide basis, which would then lead to the global rhythmic histone modifications seen here. Thus, circadian transcriptional regulators appear to be involved in the initial stages of RNAPII recruitment and initiation and the histone modifications associated with these events to set the stage for gene expression on a global scale, but additional control steps must then determine the ultimate transcriptional outputs from these sites.

In summary, we have defined the cis-regulatory network of the entire core circadian transcriptional regulatory loop on a genome scale and find a highly stereotyped, timedependent pattern of core transcription factor binding, RNAPII occupancy, RNA expression and chromatin states (Figure 5). We define three distinctive phases of the circadian cycle: 1) a poised phase in which CLOCK:BMAL1 and CRY1 bind to E-box sites in a transcriptionally silent state associated with RNAPII-Ser5P; 2) a temporally coordinated transcriptional activation phase in which RNAPII and p300 recruitment, pre-mRNA transcript expression, and $\mathrm{H} 3 \mathrm{~K} 9 \mathrm{ac}, \mathrm{H} 3 \mathrm{~K} 4 \mathrm{me} 3$ and $\mathrm{H} 3 \mathrm{~K} 27 \mathrm{ac}$ occupancy oscillate; and 3) a repression phase in which PER1, PER2 and CRY2 occupancy peaks. Circadian modulation of RNAPII recruitment and chromatin remodeling occurs on a genome-wide scale far greater than that seen previously by gene expression profiling. Thus, the circadian clock in 
the liver modulates the occupancy of RNAPII across the genome and this leads at least in part to genome-wide circadian modulation of chromatin states, which in turn, poise the genome for transcription on a daily basis to act in concert with the daily metabolic demands of the organism.

In future work, it will be important to determine how the circadian clock regulates RNAPII recruitment and initiation, as well as to explore whether pausing and pause release of RNAPII occur on a circadian basis. The widespread impact of the circadian clock on global transcription will likely impact many pathways and disease states in the organism.

\section{Acknowledgments}

J.S.T. is an Investigator at the Howard Hughes Medical Institute. Research also supported by awards from the NIH (R01AG045795).

\section{References}

1. Takahashi JS, Pinto LH, Vitaterna MH. Forward and reverse genetic approaches to behavior in the mouse. Science. 1994; 264:1724-1733. [PubMed: 8209253]

2. Lowrey PL, Takahashi JS. Genetics of circadian rhythms in mammalian model organisms. Adv Genet. 2011; 74:175-230. [PubMed: 21924978]

3. Vitaterna MH, King DP, Chang AM, et al. Mutagenesis and mapping of a mouse gene, Clock, essential for circadian behavior. Science. 1994; 264:719-725. [PubMed: 8171325]

4. Antoch MP, Song EJ, Chang AM, et al. Functional identification of the mouse circadian Clock gene by transgenic BAC rescue. Cell. 1997; 89:655-667. [PubMed: 9160756]

5. King DP, Zhao Y, Sangoram AM, et al. Positional cloning of the mouse circadian clock gene. Cell. 1997; 89:641-653. [PubMed: 9160755]

6. Gekakis N, Staknis D, Nguyen HB, et al. Role of the CLOCK protein in the mammalian circadian mechanism. Science. 1998; 280:1564-1569. [PubMed: 9616112]

7. Lowrey PL, Takahashi JS. Genetics of the mammalian circadian system: Photic entrainment, circadian pacemaker mechanisms, and posttranslational regulation. Annu Rev Genet. 2000; 34:533562. [PubMed: 11092838]

8. Preitner N, Damiola F, Lopez-Molina L, et al. The orphan nuclear receptor REV-ERBa controls circadian transcription within the positive limb of the mammalian circadian oscillator. Cell. 2002; 110:251-260. [PubMed: 12150932]

9. Busino L, Bassermann F, Maiolica A, et al. $\mathrm{SCF}^{\mathrm{Fbxl3}}$ controls the oscillation of the circadian clock by directing the degradation of cryptochrome proteins. Science. 2007; 316:900-904. [PubMed: 17463251]

10. Gallego M, Virshup DM. Post-translational modifications regulate the ticking of the circadian clock. Nat Rev Mol Cell Biol. 2007; 8:139-148. [PubMed: 17245414]

11. Siepka SM, Yoo SH, Park J, et al. Circadian mutant Overtime reveals F-box protein FBXL3 regulation of cryptochrome and period gene expression. Cell. 2007; 129:1011-1123. [PubMed: 17462724]

12. Meng QJ, Logunova L, Maywood ES, et al. Setting clock speed in mammals: the CK1 epsilon tau mutation in mice accelerates circadian pacemakers by selectively destabilizing PERIOD proteins. Neuron. 2008; 58:78-88. [PubMed: 18400165]

13. Yoo SH, Mohawk JA, Siepka SM, et al. Competing E3 ubiquitin ligases govern circadian periodicity by degradation of CRY in nucleus and cytoplasm. Cell. 2013; 152:1091-1105. [PubMed: 23452855]

14. Lowrey PL, Takahashi JS. Mammalian circadian biology: elucidating genome-wide levels of temporal organization. Annu Rev Genomics Hum Genet. 2004; 5:407-441. [PubMed: 15485355] 
15. Yoo SH, Yamazaki S, Lowrey PL, et al. PERIOD2::LUCIFERASE real-time reporting of circadian dynamics reveals persistent circadian oscillations in mouse peripheral tissues. Proc Natl Acad Sci U S A. 2004; 101:5339-5346. [PubMed: 14963227]

16. Welsh DK, Yoo SH, Liu AC, Takahashi JS, Kay SA. Bioluminescence imaging of individual fibroblasts reveals persistent, independently phased circadian rhythms of clock gene expression. Curr Biol. 2004; 14:2289-2295. [PubMed: 15620658]

17. Nagoshi E, Saini C, Bauer C, Laroche T, Naef F, Schibler U. Circadian gene expression in individual fibroblasts; cell-autonomous and self-sustained oscillators pass time to daughter cells. Cell. 2004; 119:693-705. [PubMed: 15550250]

18. Hong HK, Chong JL, Song W, et al. Inducible and reversible Clock gene expression in brain using the tTA system for the study of circadian behavior. PLoS Genet. 2007; 3:e33. [PubMed: 17319750]

19. Kornmann B, Schaad O, Bujard H, Takahashi JS, Schibler U. System-driven and oscillatordependent circadian transcription in mice with a conditionally active liver clock. PLoS Biol. 2007; 5:e34. [PubMed: 17298173]

20. Buhr ED, Yoo SH, Takahashi JS. Temperature as a universal resetting cue for mammalian circadian oscillators. Science. 2010; 330:379-385. [PubMed: 20947768]

21. Hughes ME, Hong HK, Chong JL, et al. Brain-specific rescue of Clock reveals system-driven transcriptional rhythms in peripheral tissue. PLoS Genet. 2012; 8:e1002835. [PubMed: 22844252]

22. Izumo M, Pejchal M, Schook AC, et al. Differential effects of light and feeding on circadian organization of peripheral clocks in a forebrain Bmal1 mutant. eLife. 2014:3.

23. Liu AC, Welsh DK, Ko CH, et al. Intercellular coupling confers robustness against mutations in the SCN circadian clock network. Cell. 2007; 129:605-616. [PubMed: 17482552]

24. Ko CH, Yamada YR, Welsh DK, et al. Emergence of noise-induced oscillations in the central circadian pacemaker. PLoS Biol. 2010; 8:e1000513. [PubMed: 20967239]

25. Welsh DK, Takahashi JS, Kay SA. Suprachiasmatic nucleus: cell autonomy and network properties. Annu Rev Physiol. 2010; 72:551-577. [PubMed: 20148688]

26. Ripperger JA, Schibler U. Rhythmic CLOCK-BMAL1 binding to multiple E-box motifs drives circadian Dbp transcription and chromatin transitions. Nat Genet. 2006; 38:369-374. [PubMed: 16474407]

27. Koike N, Yoo SH, Huang HC, et al. Transcriptional architecture and chromatin landscape of the core circadian clock in mammals. Science. 2012; 338:349-354. [PubMed: 22936566]

28. Lamia K, Papp S, Yu R, et al. Cryptochromes mediate rhythmic repression of the glucocorticoid receptor. Nature. 2011; 480:552-556. [PubMed: 22170608]

29. Sims RJ 3rd, Belotserkovskaya R, Reinberg D. Elongation by RNA polymerase II: the short and long of it. Genes Dev. 2004; 18:2437-2468. [PubMed: 15489290]

30. Fuda N, Ardehali M, Lis J. Defining mechanisms that regulate RNA polymerase II transcription in vivo. Nature. 2009; 461:186-192. [PubMed: 19741698]

31. Jones JC, Phatnani HP, Haystead TA, MacDonald JA, Alam SM, Greenleaf AL. C-terminal repeat domain kinase I phosphorylates Ser2 and Ser5 of RNA polymerase II C-terminal domain repeats. J Biol Chem. 2004; 279:24957-24964. [PubMed: 15047695]

32. Chapman RD, Heidemann M, Albert TK, et al. Transcribing RNA polymerase II is phosphorylated at CTD residue serine-7. Science. 2007; 318:1780-1782. [PubMed: 18079404]

33. Kim TH, Barrera LO, Zheng M, et al. A high-resolution map of active promoters in the human genome. Nature. 2005; 436:876-880. [PubMed: 15988478]

34. Guenther MG, Levine SS, Boyer LA, Jaenisch R, Young RA. A chromatin landmark and transcription initiation at most promoters in human cells. Cell. 2007; 130:77-88. [PubMed: 17632057]

35. Barski A, Cuddapah S, Cui K, et al. High-resolution profiling of histone methylations in the human genome. Cell. 2007; 129:823-837. [PubMed: 17512414]

36. Li B, Carey M, Workman JL. The role of chromatin during transcription. Cell. 2007; 128:707-719. [PubMed: 17320508] 
37. Ong CT, Corces VG. Enhancer function: new insights into the regulation of tissue-specific gene expression. Nat Rev Genet. 2011; 12:283-293. [PubMed: 21358745]

38. Rada-Iglesias A, Bajpai R, Swigut T, Brugmann SA, Flynn RA, Wysocka J. A unique chromatin signature uncovers early developmental enhancers in humans. Nature. 2011; 470:279-283. [PubMed: 21160473]

39. Creyghton MP, Cheng AW, Welstead GG, et al. Histone H3K27ac separates active from poised enhancers and predicts developmental state. Proc Natl Acad Sci U S A. 2010; 107:21931-21936. [PubMed: 21106759] 


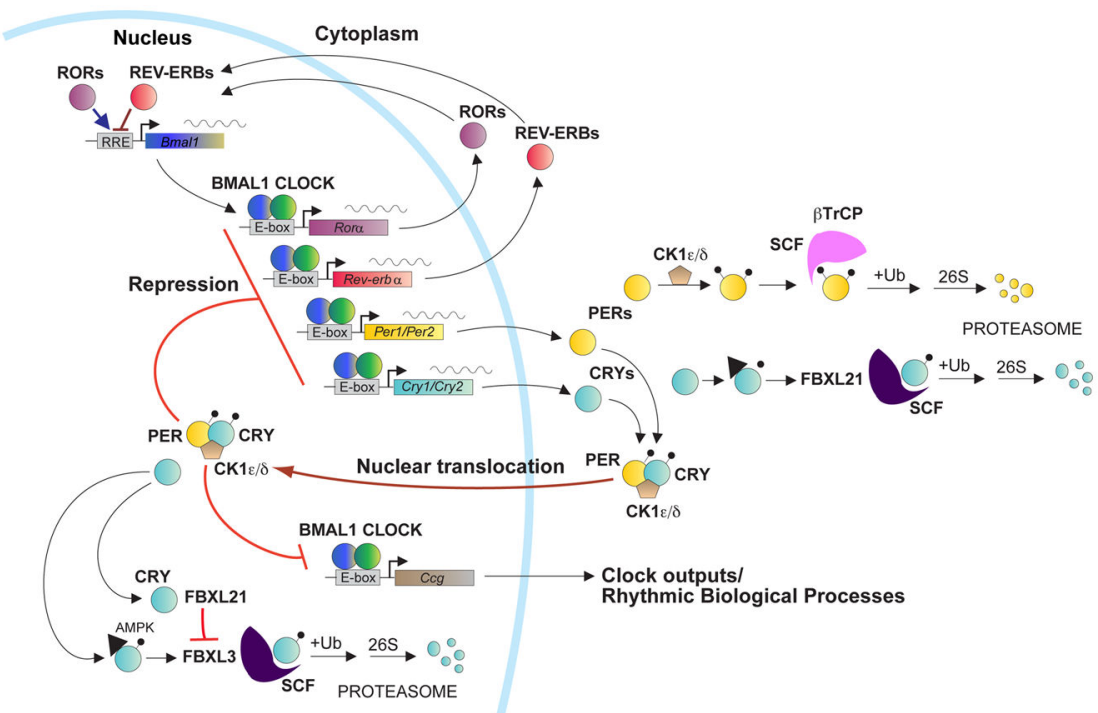

Figure 1. Model of the circadian clock in mammals

CLOCK and BMAL1 act as master transcription factors to regulate: 1) the Per and Cry genes in the core feedback loop of the clock; 2) the REV-ERB/ROR feedback loop regulating Bmall transcription; as well as 3) thousands of target genes that are clock outputs. The stability of the PER and CRY proteins are tightly regulated by E3 ubiquitin ligases in both the cytoplasm and nucleus that determine circadian period. Adapted from Mohawk et al. 2012 and Yoo et al. 2013. 


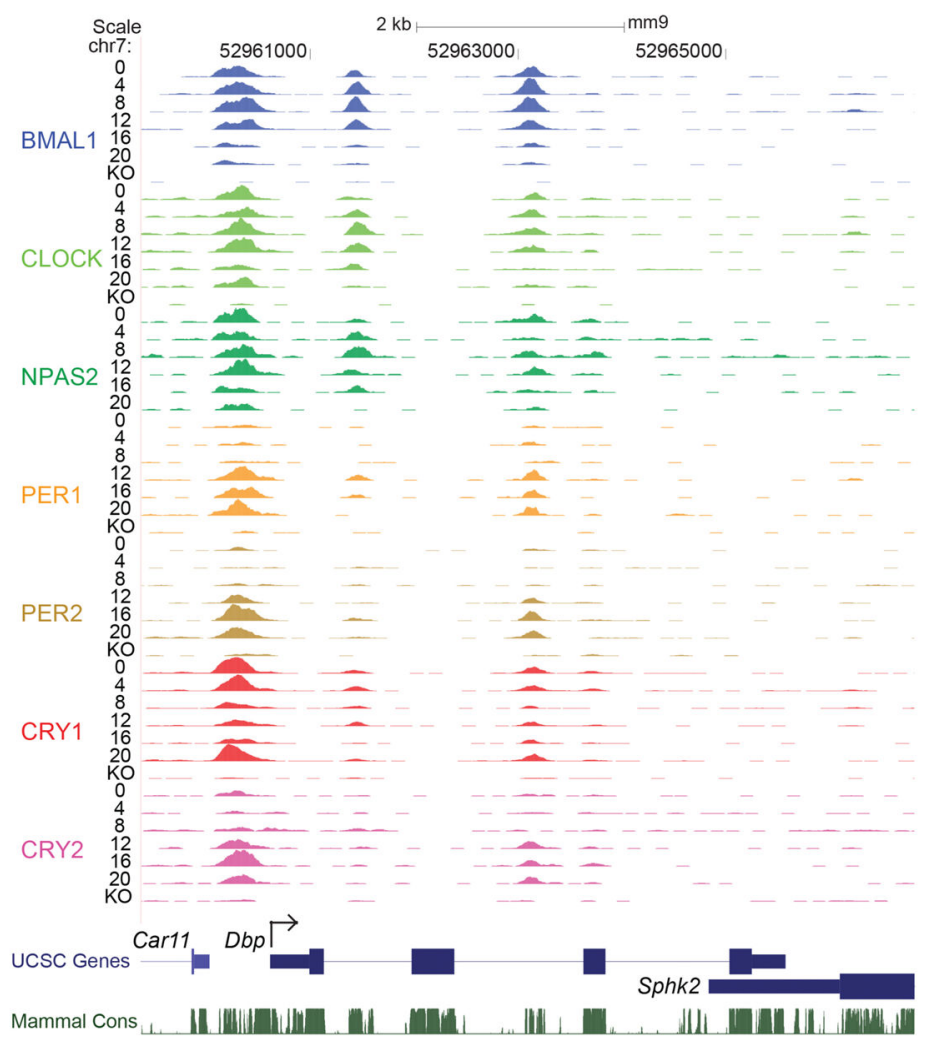

Figure 2. UCSC genome browser view of ChIP-seq profiles of circadian transcription factors at the $\mathrm{Dbp}$ gene at 6 circadian times of day

BMAL1 (blue), CLOCK (green), NPAS2 (dark green), PER1 (orange), PER2 (gold), CRY1 (red), CRY2 (pink). 0, 4, 8, 12, 16, 20 CT (hr). KO = knockout. From Koike et al. Science 2012; 338: 349-354. Reprinted with permission from AAAS. 


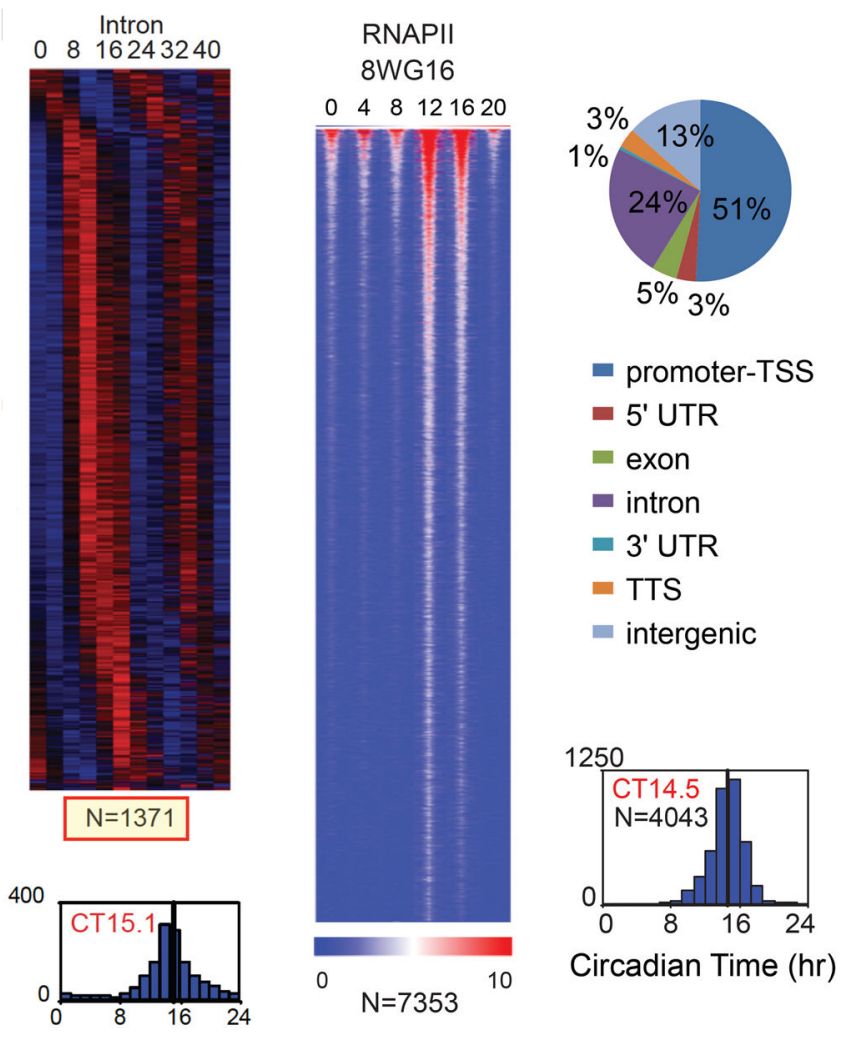

Figure 3. Heatmap views of cycling intron RNA genes (left) and RNAPII-8WG16 occupancy (right)

More than 4000 peaks had significant circadian RNAPII binding. From Koike et al. Science 2012; 338: 349-354. Reprinted with permission from AAAS. 


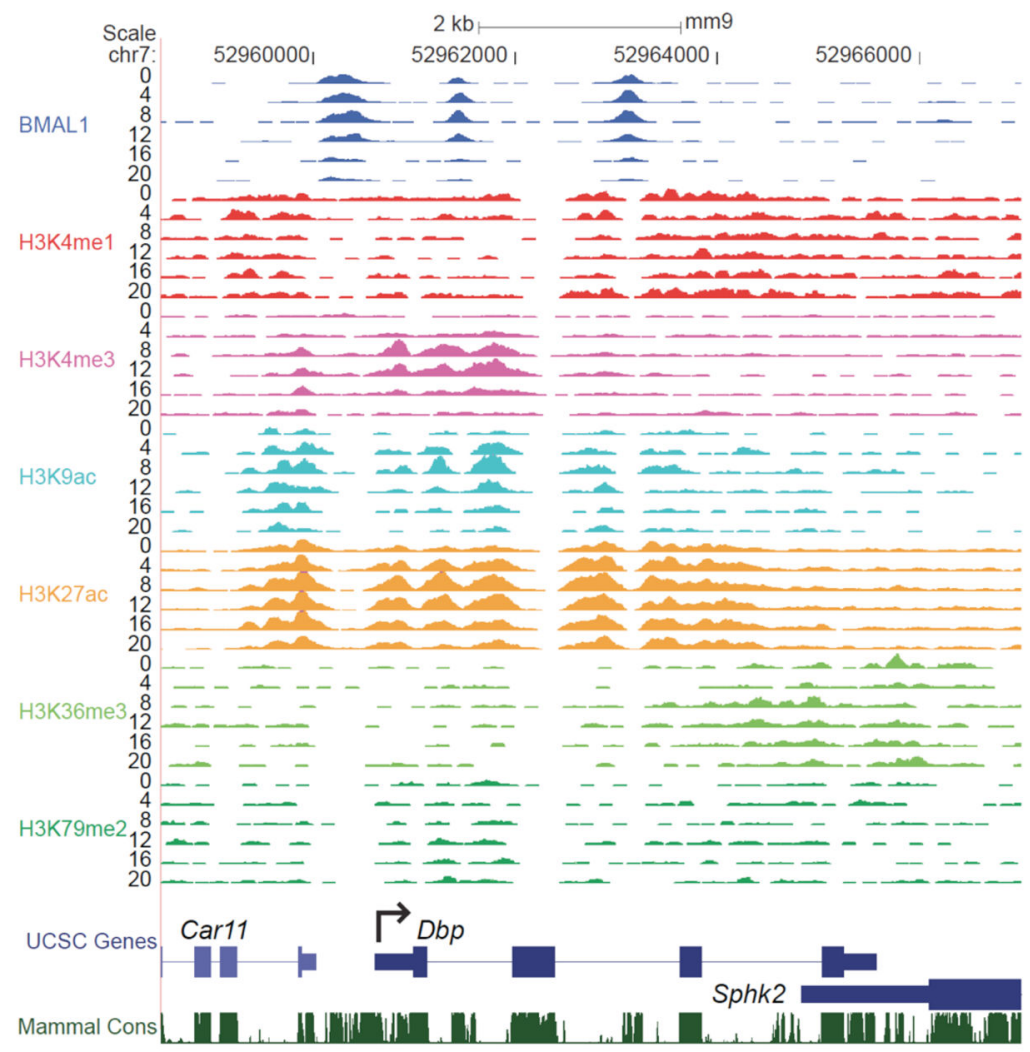

Figure 4. UCSC genome browser view of histone methylation and acetylation at the $D b p$ gene BMAL1 (blue), H3K4me1 (red), H3K4me3 (pink), H3K9ac (aqua), H3K27ac (orange), H3K36me3 (green), H3K79me2 (dark green). From Koike et al. Science 2012; 338: 349354. Reprinted with permission from AAAS. 


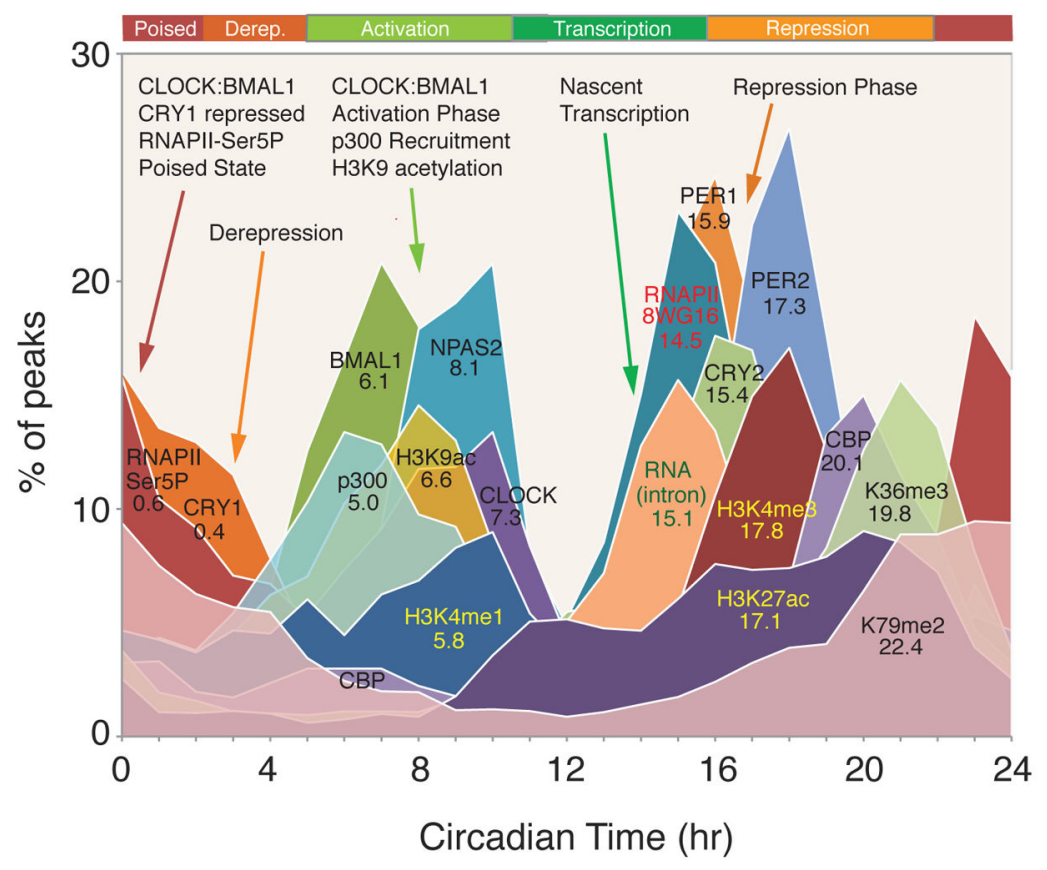

Figure 5. Circadian transcriptional landscape in the liver

Histograms show the phase distributions of each factor as a function of time of day. From Koike et al. Science 2012; 338: 349-354. Reprinted with permission from AAAS. 